\title{
Unterlassen des Mammografie-Screening als grober Behandlungsfehler
}

Das Oberlandesgericht Hamm hat in einem aktuellen Fall die Haftung eines Gynäkologen festgestellt, der eine Brustkrebserkrankung nicht rechtzeitig erkannt hatte, da er bei einer Patientin im Rahmen der Krebsvorsorgeuntersuchung zu spät zu einem Mammografie-Screening geraten hatte (Urt. v. 12.08.2013; Az.: 3 U 57/13). Die Grundsätze dieser Entscheidung, insbesondere die Einbeziehung der Patientencompliance bei der Beurteilung eines Behandlungsfehlers, sind auch auf das Fachgebiet der Radiologie übertragbar, sodass diese zukünftig beachtet werden sollten.

\section{Sachverhalt \\ $\nabla$}

Der Gynäkologe war seit dem Jahr 1985 der behandelnde Arzt der klagenden Patientin gewesen. Als solchen suchte sie ihn zu den jährlichen Brustkrebsvorsorgeuntersuchungen auf. Daneben wurden von ihm bei der Klägerin Hormonbehandlungen, am Ende mit dem Hormonersatzpräparat „Liviella“ vorgenommen. Die Vorsorgeuntersuchungen bestanden aus einer klinischen Untersuchung sowie einer Sonografie der Brust. Im Jahr 2001 fand eine Mammografie bei einem Radiologen statt. Die darauffolgenden Krebsvorsorgeuntersuchungen umfassten eine klinische Untersuchung sowie eine Sonografie der Brust. Erst im Jahr 2010 riet der Gynäkologe - trotz eines unauffälligen Befundes - wiederum zu einer Mammografie. Dort ergab sich der Verdacht eines Mammakarzinoms, der sich bei weiteren Untersuchungen bestätigte. Die Behandlung erfolgte durch Operation und Entfernung der betroffenen Lymphknoten, anschließende Strahlen- und Chemotherapie.

Die Patientin verklagte nun den Gynäkologen vor dem Landgericht Essen auf Schadensersatz, insbesondere auch auf Schmerzensgeld in Höhe von $25.000 €$. Sie argumentierte, der Brustkrebs hätte früher erkannt werden und somit schonender behandelt werden können, wenn schon ab dem Jahr 2002 zu jährlichen Mammografien geraten worden wäre. Dies insbesondere auch, weil das für ihre Hormonbehandlungen verwendete Medi- kament „Liviella“ das Risiko des Entstehens von Brustkrebs - unstreitig - zusätzlich erhöht habe.

\section{Grober Behandlungsfehler durch Nichtvornahme der Mammografie $\nabla$}

Der Fall gelangte in 2. Instanz zum OLG Hamm. Der Senat gab der Klage der Patientin weitgehend statt. Das OLG machte dies daran fest, dass der Beklagte der Patientin bereits im Jahr 2008 zu einer Mammografie hätte raten müsse, da dies die einzige sichere Methode zur Senkung des Mortalitätsrisikos gewesen sei. Diese zu spät gegebene Empfehlung zur Durchführung der Mammografie werteten die Richter ausdrücklich als groben Behandlungsfehler. Bei einem groben Behandlungsfehler verstößt der Arzt gegen bewährte ärztliche Behandlungsregeln oder gesicherte medizinische Erkenntnisse und begeht einen Fehler, der aus objektiver Sicht nicht mehr verständlich erscheint, weil er einem Arzt schlechterdings nicht unterlaufen darf.

\section{Kriterien für einen groben Be- handlungsfehler $\nabla$}

Die Grobheit des Behandlungsfehlers wurde daran festgemacht, dass die Mammografieuntersuchung zum einen im Jahr 2008 fest implementiert gewesen und die einzig sichere Methode zur Senkung des Mortalitätsrisikos bei Brustkrebs gewesen sei. Weiterhin sei zu berücksichtigen gewesen, dass die Patientin eine hohe Compliance gezeigt hätte, regelmäßig die Vorsorgetermine wahrnahm und dadurch zum Ausdruck brachte, dass es ihr ,auf die Minimierung jedweden Brustkrebsrisikos ankam“. Zudem sei ihr das Homonersatzpräparat „Liviella“ verordnet worden, dass geeignet gewesen sei, das Brustkrebsrisiko zu erhöhen. Soweit der Arzt einer Patientin unter diesen Umständen nicht dazu rate, sich einer einfachen, relativ risikolosen und von der Fachgesellschaft eindeutig empfohlenen Vorsorgeuntersuchung zu unterziehen, stelle dies einen groben Behandlungsfehler dar.

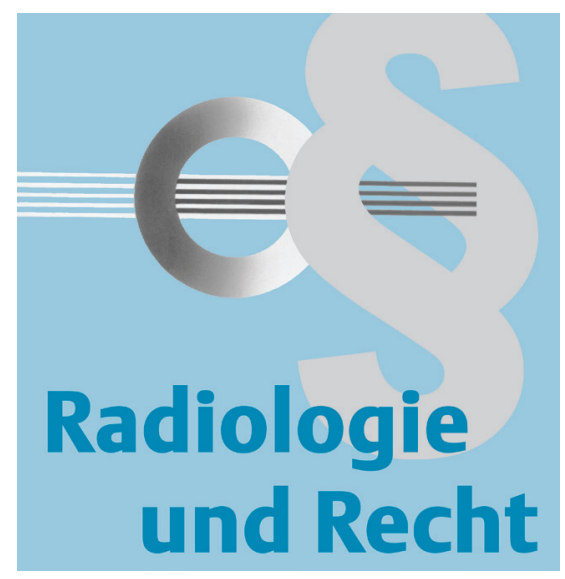

\section{Generelle Eignung zur Schadensentstehung ausreichend $\nabla$}

Die Feststellung eines groben Behandlungsfehlers hat Auswirkungen hinsichtlich des Ursachenzusammenhangs zwischen Fehler und Schaden. Es erfolgt eine Beweislastumkehr zu Lasten des behandelnden Arztes, was bedeutet, dass der Arzt nachweisen muss, dass sein Fehler nicht für den Schadenseintritt verantwortlich gewesen ist. Der Patient muss nur eine generelle Eignung des Behandlungsfehlers für die Schadensverursachung nachweisen. Dabei geht es um die bloße Möglichkeit, „nahegelegen oder wahrscheinlich machen muss der Fehler den Schaden nicht." (BGH, Urt. v. 08.01.2006; Az.: VIZR 118/06). Dabei können nach dieser Rechtsprechung andere Ursachen sogar wahrscheinlicher für die Schadenszufügung sein.

Nach diesen Grundsätzen stellte auch das OLG Hamm vorliegend einen groben Behandlungsfehler fest. Der Sachverständige habe deutlich gemacht, dass durch eine rechtzeitige Mammografie, der Tumor im Jahre 2008 rechtzeitig erkannt worden sei und hätte behandelt werden können. Es sei davon auszugehen, dass bei einer Diagnose im Jahr 2008, zu einem Zeitpunkt, an dem sich noch keine Metastasen gebildet hätten, die Operation weniger belastend gewesen wäre und die Patientin keiner Chemotherapie bedurft hätte. Zudem sei die 5-Jahres-Überlebensrate höher gewesen. Eine Tumorentdeckung zu diesem Zeitpunkt sei dabei, so der Sachverständige, auch nicht unwahrscheinlich gewesen. Den dann notwendigen Gegenbeweis, dass die gleichen Beeinträchtigungen bereits vor dem vom Gericht identifizierten Zeitpunkt im Jahr 2008 existierten und eine Behandlung notwendig gemacht hät- 
ten, konnte der Arzt naturgemäß nicht führen.

\section{Keine Aussage über Behandlungs- fehler vor Einführung des Mam- mografie-Screening \\ $\nabla$}

Allerdings schränkte das Gericht ein, dass für den Zeitraum vor dem Jahr 2008 keine medizinisch validen Aussagen gemacht werden könnten, ob der Tumor bereits zu diesem Zeitpunkt entdeckt worden wäre und ob dies ein grober Behandlungsfehler sei, weil das Mammografie-Screening „noch nicht so fest implementiert war.“ Infolgedessen war für die Schadensberechnung der Zeitpunkt ab dem Jahre 2008 zugrunde zu legen.

\section{Konsequenzen der Entscheidung für die Praxis}

Zum einen macht das Urteil des OLG Hamm noch einmal deutlich, zu welchen Konsequenzen ein grober Behandlungsfehler führen kann. Danach kann schon ein geeignetes Verhalten und eine nicht ganz unwahrscheinliche Möglichkeit eines Schadens ausreichen, den Kausalitätsnachweis zu führen, wenn der Arzt ihn nicht entkräften kann.

Ein grober Behandlungsfehler liegt dann vor, wenn ein Verstoß gegen elementare medizinische Erkenntnisse oder elementare Behandlungsstandards vorliegt. Nicht ausreichend ist nur eine Fehlentscheidung in mehr oder weniger aussichtsloser Lage (vgl. BGH, Urt. v. 25.10.2011; Az.: VI ZR 139/10).

Beachtenswert ist, dass das OLG das Kriterium der Patientencompliance in den Blick nimmt und diese bei der Frage nach den Behandlungsstandards einbezieht. Wenn ein Patient erkennbar an Früherkennungsmaßnahmen interessiert ist und diese - aufgrund risikohafter Disposition - besonders gewissenhaft verfolgt, kann eine Falschberatung durch den Arzt zu einem groben Behandlungsfehler führen. Dieses Tatbestandsmerkmal der Patientencompliance ist subjektiv und wird vom OLG nur in den Punkten des geäußerten Interesses des Patienten und einer risikohaften Disposition näher konkretisiert.

Zudem muss berücksichtigt werden, inwieweit die jeweilige Untersuchungsmethode ggf. alternativlos ist. Das bedeutet, es ist der Blick darauf zu richten, inwieweit schon eine feste Implementierung der jeweiligen Untersuchung vorliegt. Sollte dies wie vorliegend bei dem Mammografie-Screening der Fall sein, ist dies ein Kriterium dafür, dass diese diagnostische Maßnahme bei entsprechender Indikation auch angewandt werden muss.

Folge des groben Behandlungsfehlers ist die Umkehrung der Beweislast hinsichtlich der Kausalität zu Lasten des behandelnden Arztes. Konkret wird dann für den Kausalitätsnachweis zwischen Fehler und Schaden nur noch eine generelle, nicht einmal wahrscheinliche Eignung des Fehlers zur Schadensentstehung gefordert. Es ist darauf zu achten, dass selbst der Nachweis einer größeren Wahrscheinlichkeit einer anderen Ursache nicht genügt, um den Kausalitätsnachweis zu entkräften. Vielmehr müsste jegliche Ursächlichkeit im konkreten Fall äußerst unwahrscheinlich, ein ander Verstoß gegen weitere Sorgfaltspflichten vorliegend ursächlich oder gar der Patient selbst in gleicher Form für die Schadensverursachung verantwortlich sein. Die Folgen eines groben Behandlungsfehlers sind daher für den Arzt gravierend, da er sich faktisch nicht mehr exkulpieren kann.

Im Rahmen der Schadensbemessung kommt es zudem nach dem Urteil des OLG nicht nur auf den Zeitpunkt der Erkennbarkeit, sondern auch auf den Zeitpunkt der Implementierung der Untersuchungsmethode an.

Abschließend bleibt festzustellen, dass auch der Radiologe in Zukunft insbesondere die Patientencompliance bei der ärztlichen Entscheidung, auch bei Vorsorgeuntersuchungen wie dem Mammografie-Screening berücksichtigen muss, lege artis zu handeln. Dies gilt umso mehr, wenn Anhaltspunkte dafür bestehen, dass es dem Patienten erkennbar auf eine lückenlose Vorsorge ankommt oder dieser über eine entsprechende Disposition verfügt, wie vorliegend das geäußerte Interesse des Patienten an der Untersuchung oder ein z.B. durch Medikamente verursachtes erhöhtes Risiko.

RA Dr. Peter Wigge, Fachanwalt für Medizinrecht

Lic. iur. can. Urs Fabian Frigger, Rechtsanwalt

Rechtsanwälte Wigge

Scharnhorststr. 40

48151 Münster

Telefon: (0251) 53595-0

Telefax: (0251) 53595-99

E-Mail: kanzlei@ra-wigge.de www.ra-wigge.de 